\title{
Non-Linear Anisotropic Diffusion for Memory-Efficient Computed Tomography Super-Resolution Reconstruction
}

\author{
Khaled Abujbara * Ramzi Idoughi * Wolfgang Heidrich \\ KAUST \\ Thuwal, Saudi Arabia \\ [first name].[family name] dkaust.edu.sa
}

\begin{abstract}
Despite the impressive performance of Computed Tomography (CT) hardware, there is still a need to push the boundaries of the CT spatial resolution. Super-resolution techniques have been widely used in computer vision to enhance the resolution of $2 D$ and $3 D$ images. They have also been introduced to improve the CT volume resolution. In this work, we propose a flexible framework that produces a higher-resolution $3 D$ volume from low-resolution $2 D$ projections. This framework can be applied to any $C T$ data regardless of the original physical scale and regardless of the target application. It is based on regularization by denoising (RED) approach, where a Non-Linear Anisotropic Diffusion filter is used as the denoiser. We demonstrate our framework on both simulated and captured data, and show good quality reconstruction and a huge memory-footprint improvement in comparison to the state-of-the-art algorithm.
\end{abstract}

\section{Introduction}

$\mathrm{X}$-ray computed tomography $(\mathrm{CT})$ is a non-destructive imaging tool that reveals the internal structure of the scanned objects. It is based on reconstructing 3D density volumes representing the objects from a set of $2 \mathrm{D}$ projection images, acquired from different angles. X-ray CT has applications in numerous fields such as medicine and biology, security, and material and geosciences [49, 22, 36, 6, 11]. In computer vision, several algorithms and techniques have been proposed to reconstruct the density volumes under different acquisition scenarios including sparse views, discrete tomography, and dynamic tomography [2, 48, 3, 61, 62, 5]. In addition, several contributions have been made to better exploit the CT output in many computer vision tasks like segmentation, denoising, detection, rendering or superresolution $[12,45,17,64,60]$.

\footnotetext{
*Both authors contributed equally.
}

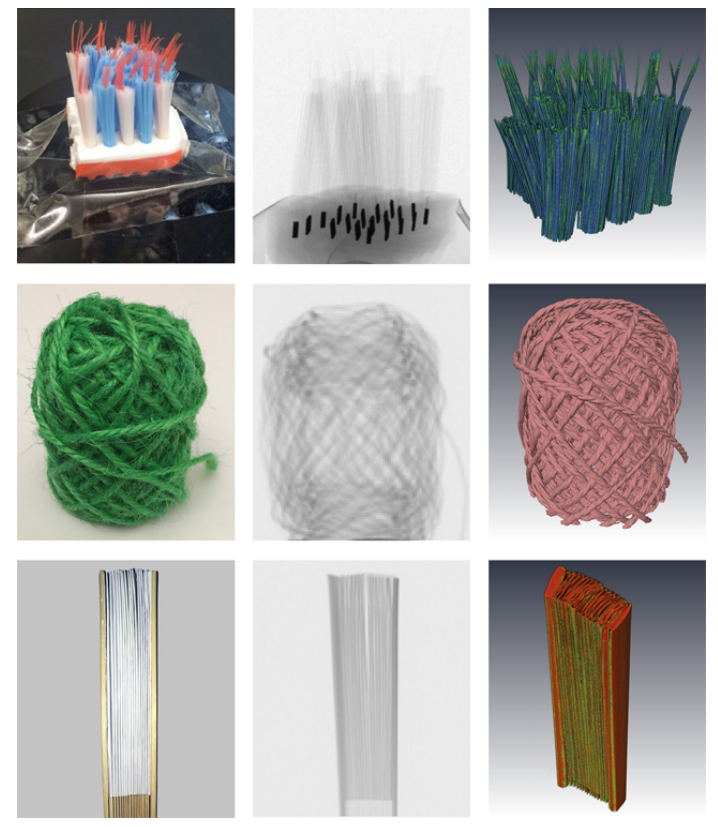

Figure 1: Our super-resolution CT reconstruction method tackles thin structures beyond the Nyquist limit of the image sensor, including thin fibrous features such as the toothbrush (top) and jute ball (middle), as well as thin surface features such as the folding paper fan (bottom). From left to right: camera photo, $\mathrm{x}$-ray image, and $3 \mathrm{D}$ reconstruction.

Since the first clinical CT device invented by Hounsfield in the early 1970s, CT hardware capabilities have seen huge improvements. Synchrotron facilities are now able to reach temporal resolutions of up to $1 \mathrm{~ms}$, and spatial resolutions of $20 \mathrm{~nm}$ or better [13]. Even lab-based CT facilities can be as fast as $12 s$ per scan and reach resolutions of $\approx 10 \mu \mathrm{m}$ [8]. However, despite these drastic improvements in peak resolution, the overall bandwidth in the acquisition system has seen much more modest improvements, which requires a delicate tradeoff between spatial resolution, temporal resolution, and field, or volume size (i.e. the physi- 
cal dimensions of the reconstructed volume). For example, while some slower X-ray sensors may have 10 megapixels, high-speed sensors may only have one megapixel or less. If such a sensor is used to investigate objects with feature sizes of $20 \mathrm{~nm}$, the total reconstruction volume would only have a diameter of ca. $20 \mu \mathrm{m}$. Besides this bandwidth limitation, the hardware costs also grow disproportionately with increasing spatial and temporal resolution.

Due to these tradeoffs, it is still of vital interest to extract the most detailed information possible from any given captured tomography dataset. Super-resolution methods help maximize spatial and temporal resolution for a given volume size while keeping the hardware costs under control. CT super-resolution methods can be classified into preprocessing, post-processing, and joint reconstruction approaches (see next section for a more detailed discussion). Of these, only joint reconstruction approaches can guarantee a strict consistency of the reconstructed volume with the raw projection data. However, joint optimization approaches come at a high cost in compute time and especially memory consumption. For example, the state-of-theart method [60] is simply not feasible for large volumes due to excessive memory usage.

In this paper, we propose a flexible framework for the super-resolution tomographic reconstruction of $3 \mathrm{D}$ volumes from lower resolution projections. This new variational framework adopts the Regularization by Denoising (RED) scheme solved with the Alternating Direction Method of Multipliers (ADMM) [42]. We use the proximal SART [3] as a solver for the tomographic reconstruction term, and the Non-Linear Anisotropic Diffusion (NLAD) method as the denoiser in the regularization step. The aim of using this denoiser is to preserve the $1 \mathrm{D}$ and $2 \mathrm{D}$ features in the $3 \mathrm{D}$ reconstructed volumes, since the denoising directions are aligned with thin 1D structures such as the fibers in the toothbrush or jute ball (Fig. 1 top, middle), or with thin 2D structures like the paper folds in a folding fan (Fig 1, bottom). In summary, the main features of our approach are:

- A joint super-resolution reconstruction for general objects, with significantly improved memory consumption at a reconstruction quality at least on par with the state-of-the-art super-resolution CT approaches.

- A RED-based super-resolution method that uses an anisotropic diffusion denoiser rather than a standard Gaussian denoiser like traditional RED frameworks (we empirically show that this denoiser respects local homogeneity and strong passivity conditions required for the convergence of RED).

- A joint optimization approach that guarantees close adherence of the reconstructed volume to the measured projection images.

\section{Related work}

Computed tomography is a well-known technique for reconstructing 3D density volumes of scanned objects, using a set of $2 \mathrm{D}$ projection images. There are two main families of tomographic reconstruction methods: transformbased approaches such as filtered back-projection [15], use the Radon transform and its inverse to provide an analytic reconstruction. These methods are fast and yield satisfying results when the tomography problem is well-posed. This is why they are still widely used by commercial CT scanners [41]. The second category are iterative methods, which are based on solving an optimization problem to obtain a solution to the reconstruction problem. The Algebraic Reconstruction Technique (ART) [21] and its variants such as the Simultaneous Algebraic Reconstruction Technique (SART) [4] are the most famous iterative reconstruction methods. They iteratively update the 3D reconstructed volume using a Kaczmarz projection scheme. The main advantage of these methods is that they may be easily combined with data regularization terms. Such framework produce better results in several ill-posed tomographic problems like sparse views [51, 27, 39], limited angles [30, 10] or dynamic tomography [61, 38, 25, 62].

With the recent rise of the deep learning, some works introduced learning-based approaches to the $\mathrm{CT}$ reconstruction to deal with ill-posed CT problems [9, 5, 7, 26, 34]. However, compared to regular images or video, CT volumes exhibit a much larger degree of domain-specific statistics, for example when comparing medical CT scans to geological rock samples or synchrotron images of fuel cells. This has so far made it impossible to find general deep network approaches that easily transfer between different application domains. For general-purpose applications modelbased iterative optimization approaches still constitute the state of the art at this time.

Super-resolution (SR) imaging is a set of algorithms that generate a higher-resolution (HR) output from lowerresolution (LR) input(s) [46, 37]. The main objective of these techniques is to overcome the limitations of the acquisition hardware, in order to improve the visualization and the post-processing of the data. Since the pioneering works [20, 14], SR has been widely used in various applications, such as medical imaging, satellite and aerial imaging, and security applications $[63,40,58,43,1]$. The SR approaches can be classified according to different criteria: The number of LR inputs (i.e. single or multiple), the application domain (i.e. spatial or frequency) and the type of the approach (i.e. interpolation-based, regularization-based or learning-based). For a comprehensive overview of the SR techniques, we invite the reader to refer to some of the numerous review papers [37, 54].

Super-resolution CT In the context of CT, the purpose of the SR techniques is to provide an HR 3D volume from 
LR 2D projections. Ideally, SR aims to resolve fine features below the Nyquist limit of the individual projections. There are three strategies of the CT SR techniques: The first strategy consists of applying the SR algorithms on the $2 \mathrm{D}$ projections as a pre-processing step of the CT reconstruction [44]. The second strategy is a post-processing step applied after the reconstruction of the 3D volume. For this strategy, the SR techniques can either be applied on the 2D slices of the volume [59,53,24], or on the 3D volume itself $[47,28,23,19]$. Both of these approaches are pipelined frameworks, where errors can accumulate between the CT reconstruction stage and the super-resolution stage, so that the final volume may actually be inconsistent with the original projection data, which may not be acceptable in some situations. Most of the recent approaches following the pre/post-processing strategies are learning-based approaches. They suffer from two main drawbacks: (1) These methods require a large database of known LR and HR image/volume pairs, which is hard to obtain even in the medical field, where the CT data are the most available. (2) To avoid this issue, the majority of these SR methods artificially construct the LR data by blurring and downsampling the captured HR data. This leads to a risk of oversimplification of the SR problem [29].

The third strategy, which we follow in this paper, consists of directly incorporating the SR module as a regularization term into an iterative $\mathrm{CT}$ reconstruction method. For objects containing a limited number of materials, SR can be obtained by imposing a discrete tomography reconstruction with a limited number of grey levels $[48,50]$. Recently, the structure tensor prior was used in order to resolve $1 \mathrm{D} / 2 \mathrm{D}$ features embedded in $3 \mathrm{D}$ reconstructed volumes [60]. This approach managed to reconstruct features beyond the Nyquist limit of the $2 \mathrm{D}$ projections and is most closely related to our work. However, it suffers from a huge memory consumption that makes it impractical to use on large volumes. Our method produces similar or better results with significantly lower memory requirements.

Nonlinear Anisotropic Diffusion (NLAD) is a class of filtering approaches that has been widely used in computer vision to denoise images while enhancing edges and local structures $[55,56,57]$. There are two main NLAD filters: Edge Enhancing Diffusion (EED) [55]; which is an adaptive steered gradient-based filter that allows smoothing along the edges while it inhibits the smoothing across them. The other main filter is the Coherence Enhancing Diffusion (CED) [56, 57]. For this second filter, the smoothness is performed along coherent flow-like structures. The NLAD filtering has been applied in several computer vision tasks like denoising [16, 35], data compression [18], super-resolution [31,33]. Nevertheless, to the best of our knowledge, NLAD has never been used as a regularization term in a super-resolution CT reconstruction framework, as we propose in the current work.

\section{Methodology}

\subsection{Variational formulation}

For a scanned object, the $N$ captured projections are obtained from the $3 \mathrm{D}$ density volume $\mathbf{x} \in \mathbb{R}^{N_{v}}$ representing the object, using the following equation:

$$
\mathbf{p}_{i}=\mathbf{A}_{i} \cdot \mathbf{x}+\mathbf{n}_{i},
$$

where $\mathbf{A}_{i} \in \mathbb{R}^{N_{p} \times N_{v}}$ and $\mathbf{n}_{i}$ are respectively the Radon transform operator and the noise distribution corresponding to the $i^{t h}$ projection $\mathbf{p}_{i} . N_{p}$ and $N_{v}$ are respectively the number of pixels in the projections and the number of voxels of the 3D volume.

The tomographic reconstruction problem is a linear inverse problem that consists of retrieving the $3 \mathrm{D}$ density volume $\mathbf{x}$, from the set of $N$ captured projections $\left(\mathbf{p}_{i}\right)_{1 \leq i \leq N}$. This can be formulated as a convex optimization problem, where the loss function is given by:

$$
\mathbf{x}^{*}=\underset{\mathbf{x}}{\operatorname{argmin}} \sum_{i=1}^{N}\left\|\mathbf{A}_{i} \cdot \mathbf{x}-\mathbf{p}_{i}\right\|_{2}^{2}+\lambda \cdot \mathcal{R}(\mathbf{x})
$$

where the first term is the data-fitting term, that penalizes the discrepancy between the reconstructed volume and the captured projections, $\mathcal{R}($.$) is regularization term that we$ will present below. $\lambda$ is the weight associated with the regularization term.

\subsection{Regularization by Denoising (RED)}

To solve the optimization problem presented in Equation 2, we follow the Regularization by Denoising (RED) [42] paradigm. Given a denoiser engine $D($.$) respecting the lo-$ cal homogeneity and the strong passivity conditions (one can refer to [42] for more details), the RED approach proposes the use of a penalty term proportional to the innerproduct between the volume $\mathbf{x}$ and its denoising residual $(\mathbf{x}-D(\mathbf{x}))$. This prior is expressed as:

$$
\mathcal{R}(\mathbf{x})=\frac{1}{2} \cdot \mathbf{x}^{T}(\mathbf{x}-D(\mathbf{x}))
$$

The optimization problem in Equation 2, then becomes:

$$
\mathbf{x}^{*}=\underset{\mathbf{x}}{\operatorname{argmin}} \sum_{i=1}^{N}\left\|\mathbf{A}_{i} \cdot \mathbf{x}-\mathbf{p}_{i}\right\|_{2}^{2}+\frac{\lambda}{2} \cdot \mathbf{x}^{T}(\mathbf{x}-D(\mathbf{x})) .
$$

The advantages of this approach are the explicit separation between the data-fitting term and the prior, and the ability to introduce powerful denoisers as regularization terms without the need to differentiate them. Moreover, RED has shown state-of-the-art performances in several imaging problems. 


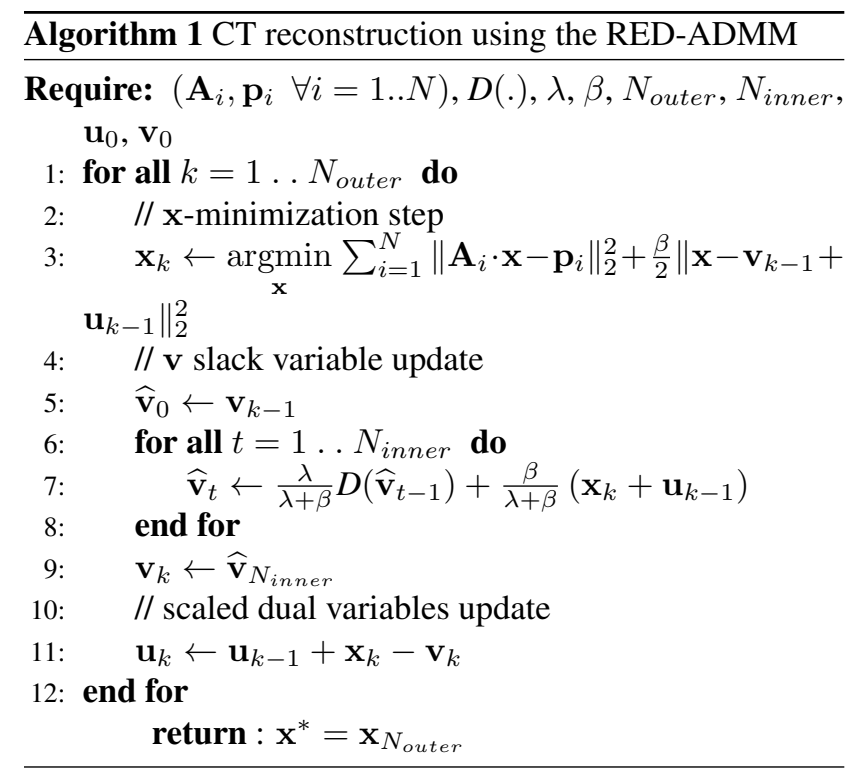

In our implementation we adopt the ADMM scheme to split and to solve the optimization problem in Equation 4. In Algorithm 1 we provide the pseudo-code of our used framework; where $\beta$ is the ADMM coefficient, $\mathbf{v}$ is the slack variable, $\widehat{\mathbf{v}}$ is a temporary variable that is used to compute the slack variable. $\mathbf{u}$ is the dual variable, $N_{\text {outer }}$ and $N_{\text {inner }}$ are respectively the number of the outer loop and the inner loop iterations. The inner loop illustrated in this pseudocode corresponds to an iterative update of the slack variable using a fixed-point strategy. With regard to the update of the density volume $\mathbf{x}$ (line 3 in Algorithm 1), we use the proximal SART algorithm [3]. The corresponding pseudocode is given in the supplement material document. Contrary to the notation in Algorithm 1, the Proximal SART algorithm does not require an explicit representation of the matrix $\mathbf{A}_{i}$. Instead, a matrix-free procedural implementation can be used to compute the projection of a given volume $\mathbf{x}$.

\subsection{Denoising by Non-linear Anisotropic Diffusion}

In this subsection we will explain the non-linear anisotropic diffusing-based denoiser $D($.$) that we use in$ our framework to denoise the volume $\mathbf{v}$ (slack variable in the Algorithm 1). The aim of introducing this denoiser is to preserve $1 \mathrm{D} / 2 \mathrm{D}$ thin features embedded in the reconstructed volume, while smoothing the homogeneous regions of the volume. Anisotropic diffusion relies, in essence, on adapting the smoothing according to the gradient's directions/orientations. The easiest structure descriptor is then the gradient of a Gaussian-smoothed version of the volume v. This gradient is defined as:

$$
\nabla \mathbf{v}_{\sigma}=\nabla\left(K_{\sigma} * \mathbf{v}\right)
$$

where $K_{\sigma}$ is a 3D Gaussian kernel, $\sigma$ is the standard deviation of the kernel, and $*$ corresponds to the convolution operator. The gradient is a structure descriptor that provide only a direction information, which is useful for edge detection, but unsuitable for the case of parallel structures. To deal with this limitation, the structure tensor $S_{\rho}\left(\nabla \mathbf{v}_{\sigma}\right)$ is often used to describe the local gradient orientations:

$$
S_{\rho}\left(\nabla \mathbf{v}_{\sigma}\right)=K_{\rho} *\left(\nabla \mathbf{v}_{\sigma} \nabla \mathbf{v}_{\sigma}^{T}\right) \quad(\rho \geq 0)
$$

Where $K_{\rho}$ is another 3D Gaussian kernel with a standard deviation equals to $\rho$. The structure tensor is a symmetric positive and semidefinite matrix. Its eigenvalues $\left(\mu_{1}, \mu_{2}, \mu_{3}\right)$ indicate the local contrast along the corresponding eigenvectors $\left(\omega_{1}, \omega_{2}, \omega_{3}\right)$, which give the local orientations of the structure. Thereafter, we consider that the eigenvalues are ordered:

$$
0 \leq \mu_{1} \leq \mu_{2} \leq \mu_{3}=\mu_{\max }
$$

There are different structure types according to the eigenvalues. The first case $\left(\mu_{\max } \gg \mu_{2} \simeq \mu_{1}\right)$ corresponds to a planar structure. The second case corresponding to a tubular structure is obtained when $\left(\mu_{\max } \simeq \mu_{2} \gg \mu_{1}\right)$. The last case occurs when the three eigenvalues are in the same order of magnitude, it corresponds to an isotropic structure. In the following we will explain how the structure tensor is used to enhance the quality of the volume $\mathbf{v}$ using coherenceenhancing anisotropic diffusion filtering approach.

The non-linear anisotropic diffusion filtering of the volume $\mathbf{v}$ consists of solving the following partial differential equation (PDE):

$$
\begin{aligned}
\frac{\partial \mathbf{w}}{\partial t} & =\operatorname{div}(\Psi \nabla \mathbf{w}) \\
\mathbf{w}_{t=0} & =\mathbf{v}
\end{aligned}
$$

Here, $\mathbf{w}$ is a volume that will be modified at each iteration by applying the diffusion tensor $\Psi$. w is initially equal to $\mathbf{v}$, the volume that we would like to denoise. The diffusion tensor $\Psi$ is defined from the structure tensor $\left(S_{\rho}\left(\nabla \mathbf{w}_{\sigma}\right)\right)$ of the volume $\mathbf{w}$. The two tensors have the same eigenvectors $\left(\omega_{1}, \omega_{2}, \omega_{3}\right)$, and the eigenvalues of the diffusion tensor are related to $\left(\mu_{1}, \mu_{2}, \mu_{\max }\right)$ as follows:

$$
\nu_{i}=\alpha+(1-\alpha) \cdot \exp \left(\frac{-C}{\left(\mu_{\max }-\mu_{i}\right)^{2}}\right) \quad i=1,2,3
$$

where $\alpha>0$ and $C>0$ are two parameters that control the strength of the diffusion filtering. The parameter $\alpha$ ensures that the diffusion tensor is always uniformly positive definite, since $\min \left\{\nu_{1}, \nu_{2}, \nu_{3}\right\}=\alpha>0$. This enforces a diffusion even for the isotropic structures of the volume. The parameter $C$ is a threshold; if $C \gg\left(\mu_{\max }-\mu_{i}\right)^{2}$ then the diffusivity $\nu_{i} \approx 1$, and we get $\nu_{i} \approx \alpha$ if $C \ll$ $\left(\mu_{\max }-\mu_{i}\right)^{2}$. 


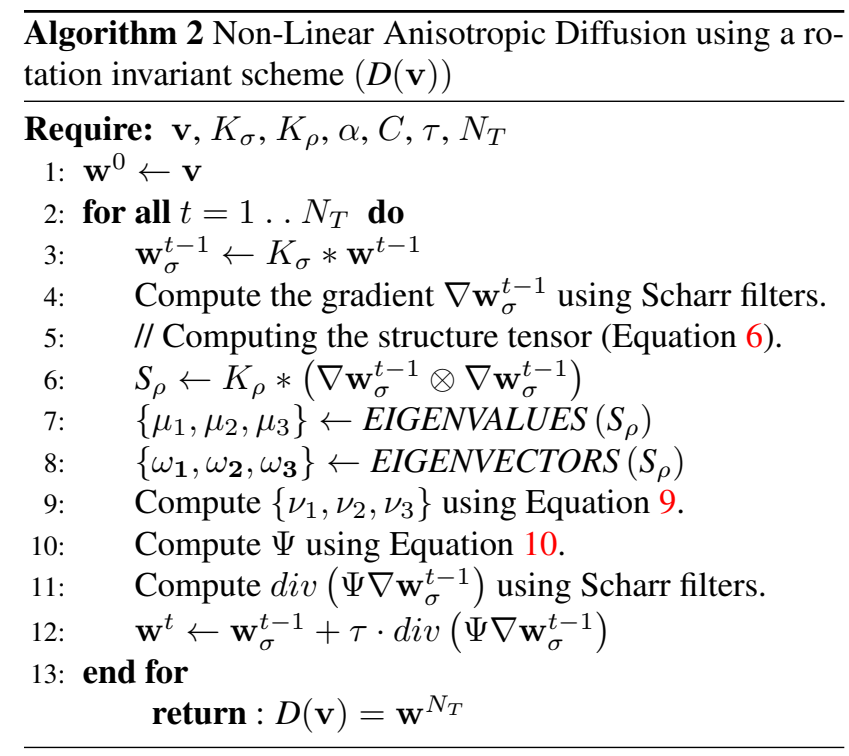

The diffusion tensor $\Psi$ smoothes mainly along the directions $\omega_{1}$ and $\omega_{2}$ with a diffusivity $\nu_{i}$ that increases with the discrepancy $\left(\mu_{\max }-\mu_{i}\right)$. The diffusion tensor is defined as follows:

$$
\Psi=\left[\begin{array}{lll}
\omega_{\mathbf{1}} & \omega_{\mathbf{2}} & \omega_{\mathbf{3}}
\end{array}\right]\left[\begin{array}{ccc}
\nu_{1} & 0 & 0 \\
0 & \nu_{2} & 0 \\
0 & 0 & \nu_{3}
\end{array}\right]\left[\begin{array}{l}
\omega_{\mathbf{1}}^{T} \\
\omega_{\mathbf{2}}^{T} \\
\omega_{\mathbf{3}}^{T}
\end{array}\right]
$$

To apply the NLAD filter on the target volume $\mathbf{v}$, we use a discretization version of the PDE in Equation 9. The temporal derivative is discretized using a backward difference, while the divergence term is discretized following a 3D rotation-invariant scheme $[57,32]$, in order to better smooth curved-like structure. Specifically, the spatial derivative $\partial_{x}$, $\partial_{y}$ and $\partial_{z}$ are implemented using a Scharr filter, which provides similar results to using a $5 \times 5 \times 5$ stencil [32].

The pseudo-code in Algorithm 2 summarizes the main steps of the non-linear anisotropic diffusion filter that we use as a denoiser in our RED-based framework.

The RED framework is proven to converge if denoiser satisfies two conditions, local homogeneity and strong passivity. These conditions can be validated empirically with numerical experiments. As we show in the supplemental material, the NLAD filter satisfies both conditions, which ensures convergence of our approach.

\section{Results and discussion}

Baselines: In this section, we compare our approach to three baseline reconstruction techniques. The first baseline is the Simultaneous Algebraic Reconstruction Technique (SART) [4], since this iterative tomographic reconstruction method produces decent results even when using a low number of projections. The second baseline is a primaldual proximal framework that uses the SART as a solver for the data-fitting term and the Anisotropic Total Variation as regularizer (PSART-ATV) [3, 52]. The last baseline is another primal-dual proximal framework, which uses the Structure Tensor Prior (PSART-STP) as a regularizer [60]. This approach is the state-of-the-art of the joint reconstruction approaches for super-resolution. For all the compared methods, the volume is initialized with 0 , and then is reconstructed from downsampled cone-beam projections uniformly distributed over a circle. These projections are obtained by downsampling the captured tomograms, with suitable factors for each dataset.

Parameters: The experiments are conducted on a computer with 512 GB RAM and a $2.80 \mathrm{GHz}$ Intel Xeon Gold 6242 Processor. The implementation of our framework is done in $\mathrm{C}++$, and the computations are parallelized using OpenMP over 32 cores.

A wide range of experiment parameters are tested, and those of the best performance were selected. We provide the parameters used for the three baseline methods in the supplement material. For our method, some parameters were not the same for all the datasets; they are shown in Table 1. The remaining parameters specific to the denoiser were similar for all the reconstructions: one inner iteration $\left(N_{\text {inner }}\right)$ in Algorithm 1 was good enough for the reconstructions, similarly only one iteration in the diffusion process $\left(N_{T}\right)$ in Algorithm 2 was performed, with a time step $(\tau)$ equal to 1 . Finally, the threshold $(C)$ was set to $1 e-10$.

Table 1: Parameters used to run our framework for the simulated and real data.

\begin{tabular}{l|cccccc}
\hline Parameters & FZP2 & FZP4 & FZP8 & Toothbrush & Ropeball & Fan \\
\hline \hline Main loop iterations: $N_{\text {outer }}$ & 25 & 25 & 12 & 12 & 6 & 7 \\
Nested SART iterations & 3 & 3 & 3 & 2 & 2 & 3 \\
Prior parameter: $\lambda$ & 2 & 2 & 2 & 2 & 5 & 5 \\
ADMM parameter: $\beta$ & 10 & 10 & 10 & 10 & 5 & 10 \\
Diffusivity: $\alpha$ & $1 \mathrm{e}-3$ & $1 \mathrm{e}-3$ & $1 \mathrm{e}-3$ & $1 \mathrm{e}-3$ & $1 \mathrm{e}-1$ & $1 \mathrm{e}-3$ \\
\hline \hline
\end{tabular}

Reference volumes: For all the dataset that we used in our quantitative validation, we reconstruct a reference volume. For the simulated data, the reference is a ground truth volume simulated at the super-resolution scale. For real capture data, a ground truth is hard to come by, so we reconstruct a reference volume at the original resolution, and then evaluate the different methods by also reconstructing from downsampled projections. The reference volume is reconstructed using the PSART-ATV framework to avoid a bias towards our new method.

\subsection{Synthetic results}

First, we validate the super-resolution performance of our framework using a synthetic 3D Fresnel Zone Plate 

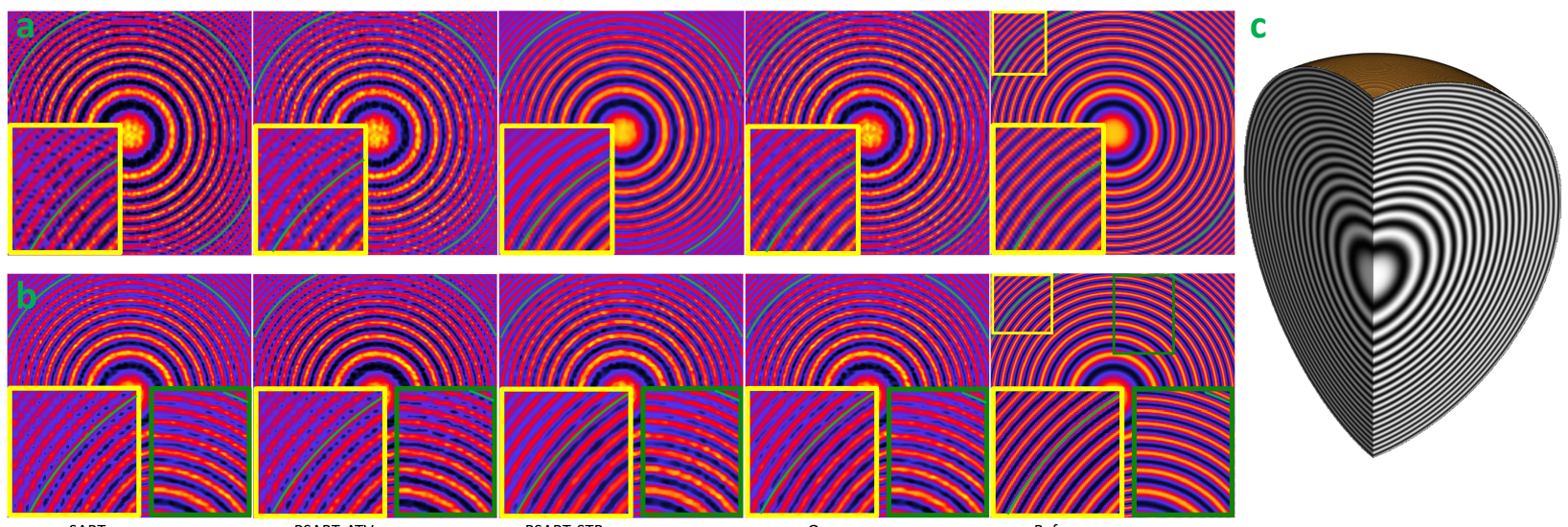

Ours

Reference

Figure 2: Comparison results for the reconstruction of the Fresnel Zone Plate using 2D cross-sections. (a) FZP2: reconstruction with a $\times 2$ super-resolution. (b) FZP4: reconstruction with a $\times 4$ super-resolution. (c) $3 \mathrm{D}$ rendering of the ground truth FZP8.
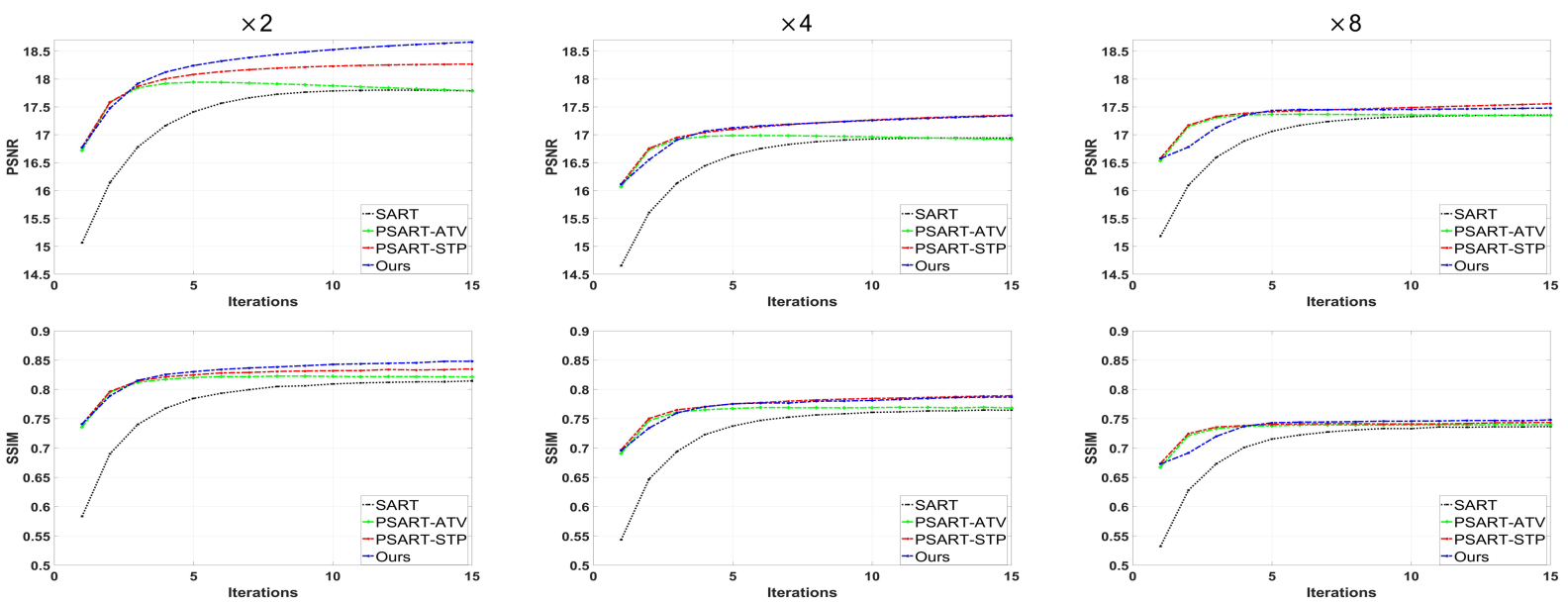

Figure 3: Comparison of the PSNR (top row) and the SSIM (bottom row) results for FZP2, FZP4, and FZP8 respectively.

(FZP). This dataset is often used in 2D/3D super-resolution applications, since the distance between the concentric circles/spheres is gradually decreasing until going under the Nyquist limit. In our experiments, we generate a 3D volume with a size of $1024^{3}$ (see Figure 2-(c)). Then we simulate 180 cone-beam projection images, with an angular spacing of $2^{\circ}$. We add a Gaussian noise $(\sigma=2)$ to the projections before downsampling them by factor 8 , using a bicubic interpolation. From the obtained projections $(128 \times 128 \times 180)$, we reconstruct volumes with three different super-resolution factors: $\times 2, \times 4$ and $\times 8$. These volumes have respectively the following sizes: FZP2 $\left(256^{3}\right)$, FZP4 $\left(512^{3}\right)$ and FZP8 $\left(1024^{3}\right)$. The initial simulated volume is used as the ground truth when comparing the reconstructed volumes at the scale FZP8. For the two other scales, we use a reference obtained by downsampling the initial volume by 2 and 4. In Figure 2-(a) and (b), we il- lustrate 2D cross-sections from the results of the different reconstruction methods. The first line corresponds to the FZP2 scenario, while the second line presents the case of FZP4. Please refer to the supplement for the FZP8 reconstructions. We also show in Figure 3 the obtained PSNR and the SSIM over the iterations, for the compared methods and for the three super-resolution scales.

From Figure 2-(a) and (b), we observe that for all methods the quality of the reconstruction gets closer towards the center. Except for the PSART-STP, which yields a smoother, less blocky results in the FZP2 case. For this same scenario, we see clearly that our approach and PSART-STP achieve better, more consistent reconstruction of the outer rings compared to SART and PSART-ATV, especially for frequencies above the Nyquist limit (green circle). This same observation can also be made in the FZP4 case. Moreover, we notice that there are aliasing artifacts 
in the middle-top region (green zoomed box) for the three baseline approaches, while our framework manages to reduce these artifacts. The analysis of the PSNR and SSIM in Figure 3 shows a better performance of our framework in the case of FZP2, where these metrics are as follows: SART $(17.79,0.8130)$, PSART-ATV $(17.94,0.8187)$, PSARTSTP $(18.24,0.8359)$, Ours $(\mathbf{1 8 . 7 9}, \mathbf{0 . 8 5 4 2})$. In the case of FZP4 and FZP8, our approach has similar quantitative results to the state-of-the-art approach PSART-STP. Nevertheless, Table 2 illustrates a faster convergence time and a dramatic decrease in the memory footprint of our method in comparison to PSART-STP.

Table 2: Parameters used to run our framework for the simulated and real data: Time elapsed (hh:mm:ss) / Memory (GB).

\begin{tabular}{c|cccccc}
\hline Method & FZP2 & FZP4 & FZP8 & Toothbrush & Ropeball & Fan \\
\hline \hline SART & $00: 10: 00$ & $01: 16: 00$ & $04: 58: 00$ & $03: 07: 51$ & $00: 23: 19$ & $00: 30: 36$ \\
& 0.53 & 4 & 32 & 8.47 & 0.88 & 2.12 \\
\hline PSART-ATV & $00: 30: 44$ & $03: 43: 30$ & $24: 14: 00$ & $03: 43: 52$ & $00: 55: 23$ & $00: 52: 58$ \\
& 0.71 & 5.5 & 48 & 12.57 & 1.17 & 2.84 \\
\hline PSART-STP & $01: 29: 10$ & $11: 03: 45$ & $41: 24: 04$ & $07: 44: 11$ & $01: 13: 00$ & $01: 34: 58$ \\
& 5.8 & 47 & 346 & 122.88 & 10.58 & 18.43 \\
\hline Ours & $01: 04: 34$ & $08: 09: 10$ & $28: 49: 12$ & $06: 20: 23$ & $01: 02: 30$ & $01: 12: 05$ \\
& 1.6 & 10.6 & 82 & 22.13 & 1.98 & 4.18 \\
\hline \hline
\end{tabular}

\subsection{Real world results}

Toothbrush Fibers: The first dataset (Toothbrush) is a good example for tubular structures embedded in the 3D volume. This dataset was already used in [60] to validate the PSART-STP method. However, we made the dataset more challenging by downsampling the 360 projections by factor 4 . Then we apply the 4 reconstruction methods with a super-resolution factor 4 . Note that we reconstructed only a Region Of Interest (ROI) of the scanned volume in order to focus on the fibers features while reducing the computational times. In Figure 4, the reference reconstruction retrieves fibers with different intensities between the center and the border. Our approach is the only one that reproduces such distribution among the compared methods. Also, it has more advantage in separating the edges of the fibers since it smoothes along the homogeneous regions in an anisotropic fashion, outperforming all the baseline methods.

Jute Ribbon Ropeball: The second dataset is another example of 1D features embedded in the volume. Nevertheless, in this case the bundles of thin and twisted fibers form short and curved cylindrical features. The 4 compared methods are here challenged to retrieve the thin fibers without being merged and also separating the bundles. For this data 720 projections were captured at a resolution of $1910 \times 1524$. These projections were downsampled using a factor 12, in order to be used as input of the super-resolution reconstructions. In this experiment, the super-resolution factor was set to 3, and by selecting a ROI the size of the output volumes is $290 \times 350 \times 300$. The results shown in Figure 5, point out that our framework has a better separation of the fiber features than the three baselines. Thus, the arrows in the green and blue boxes illustrate an almost clear separation between the structures with our reconstruction, while with other methods these structures tend to be merged together. For the red and the yellow zoomed boxes, two fibers are merged together for all methods. However, with our reconstruction, we can see a slightly darker line representing the edge between the two fibers (see arrows). Note that in the top-right side of the yellow box, the separation between the fibers is better reproduced with other methods than ours.

Foldable Fan: Finally, the last dataset represents a foldable Fan, which is a good example of 2D planer sheet structures embedded in a 3D volume. This data was scanned using 3000 projections that were all used for the reconstruction of the reference volume. However, only 187 downsampled projections were used as input of the super-resolution reconstructions. The results presented in Figure 6 show that our framework obtains smoother but simultaneously sharper results while being able to split the individual folds (see close-up views in mid subfigures).

In summary, for both simulated and real scanned data, our reconstruction framework gives better results than the three baseline methods, in terms of both quantitative (PSNR and SSIM) and qualitative comparisons (visualization of the volume), allowing for super-resolved reconstruction of thin structures. Moreover, Table 2 shows faster convergence time and a significant memory consumption improvement in comparison to the state-of-the-art PSART-STP.

\section{Conclusions and future work}

In this work, we present a flexible framework for joint super-resolution CT reconstruction based on regularizationby-denoising. A Non-Linear Anisotropic Denoiser is leveraged as a regularizer, alongside with the Simultaneous Algebraic Reconstruction Technique (SART). Our method moderately outperforms the general state-of-the-art superresolution CT reconstruction methods, in both quality and time, while significantly reducing the memory footprint, making it tractable to reconstruct larger volumes. These results were confirmed with both simulated and real objects.

Although our work mainly focuses on static object imaging, the presented method could potentially be helpful for various tasks such as dynamic fluid imaging, carbon fibers composite under fluid tension, and nano X-ray tomography reconstruction. Other future directions include a multi-scale scheme to achieve higher reconstruction resolutions, and 


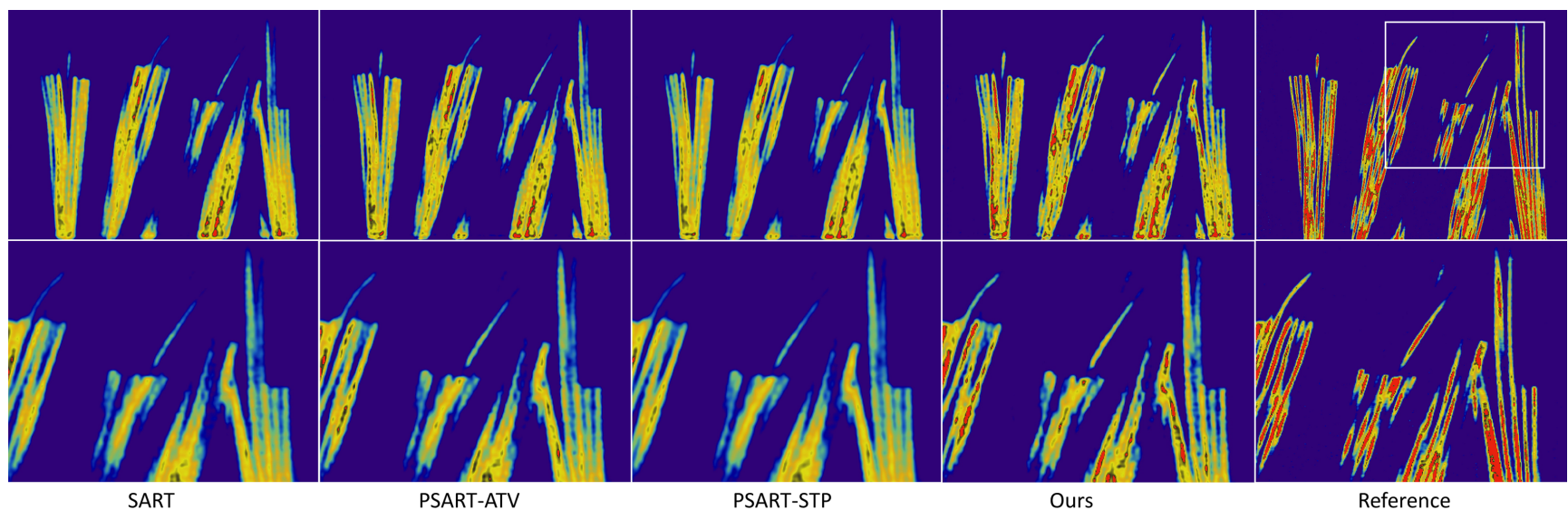

Figure 4: Representative slice visualization (top row) and its close-up view (bottom row) for the Toothbrush dataset, reconstructed respectively by SART, PSART-ATV, PSART-STP, Ours, and the reference volume.

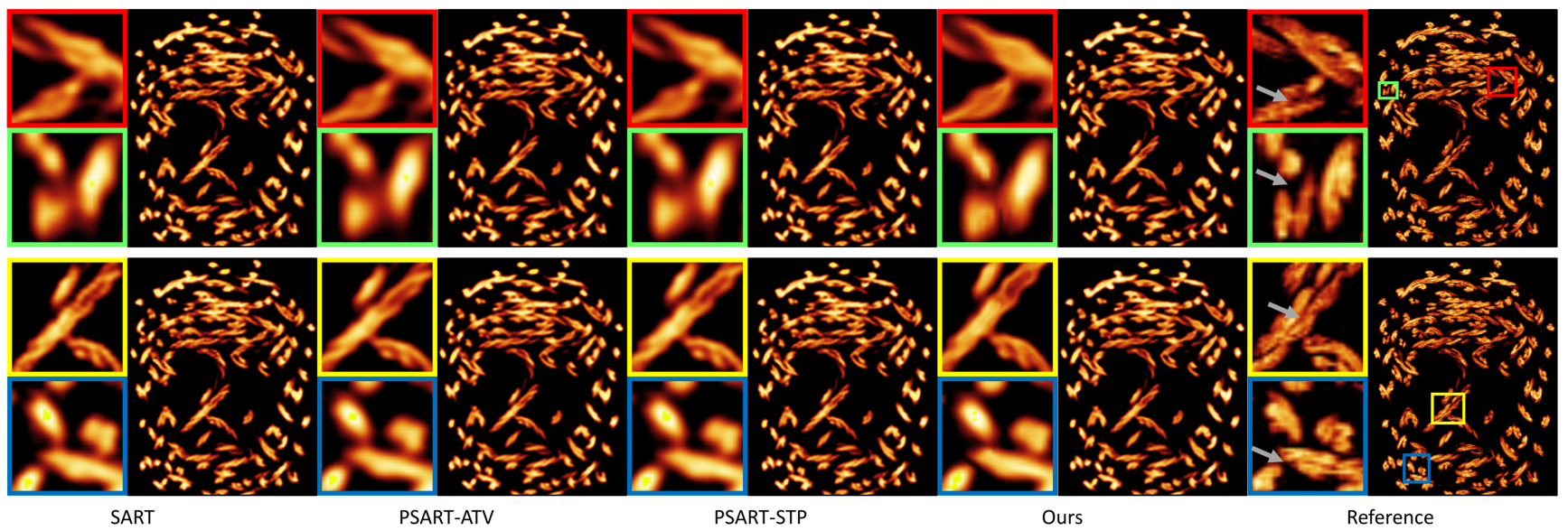

Figure 5: Representative slice visualization and its related 4 close-up views for the Robeball dataset, reconstructed respectively by SART, PSART-ATV, PSART-STP, Ours, and the reference volume.

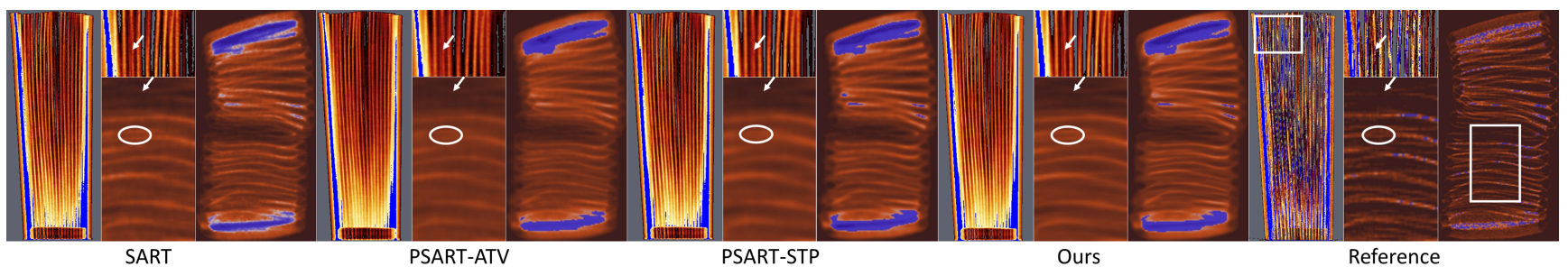

Figure 6: Representative slices visualization for the foldable Fan, in the axial and the sagittal planes with a close-up views for respectively: reconstruction results of SART, PSART-ATV, PSART-STP, Ours and the reference volume.

optimized implementation for the structure tensor computation through a sliding window kernel.

\section{Acknowledgment}

This work was supported by KAUST as part of the VCC Competitive Funding. The authors would like to thank the anonymous reviewers for their insightful comments, and Ran Tao for helping with the data collection. We also thank Guangming Zang, Prem Chedella and Moetaz Abbas for constructive discussions. 


\section{References}

[1] Muhannad Almutiry. Wideband tomographic superresolution radar image. IEEE Sensors Journal, 20(3):12081216, 2019. 2

[2] Christopher V Alvino and Anthony J Yezzi. Tomographic reconstruction of piecewise smooth images. In Proceedings of the 2004 IEEE Computer Society Conference on Computer Vision and Pattern Recognition, 2004. CVPR 2004., volume 1, pages I-I. IEEE, 2004. 1

[3] Mohamed Aly, Guangming Zang, Wolfgang Heidrich, and Peter Wonka. TRex: A tomography reconstruction proximal framework for robust sparse view X-ray applications. arXiv preprint arXiv:1606.03601, 2016. 1, 2, 4, 5

[4] Anders H Andersen and Avinash C Kak. Simultaneous algebraic reconstruction technique (SART): a superior implementation of the ART algorithm. Ultrasonic Imaging, 6(1):81-94, 1984. 2, 5

[5] Rushil Anirudh, Hyojin Kim, Jayaraman J Thiagarajan, K Aditya Mohan, Kyle Champley, and Timo Bremer. Lose the views: Limited angle CT reconstruction via implicit sinogram completion. In Proc. CVPR, pages 6343-6352, 2018. 1,2

[6] Sébastien Brisard, Marijana Serdar, and Paulo JM Monteiro. Multiscale X-ray tomography of cementitious materials: A review. Cement and Concrete Research, 128:105824, 2020. 1

[7] Tatiana A Bubba, Gitta Kutyniok, Matti Lassas, Maximilian März, Wojciech Samek, Samuli Siltanen, and Vignesh Srinivasan. Learning the invisible: A hybrid deep learningshearlet framework for limited angle computed tomography. Inverse Problems, 35(6):064002, 2019. 2

[8] Tom Bultreys, Marijn A Boone, Matthieu N Boone, Thomas De Schryver, Bert Masschaele, Denis Van Loo, Luc Van Hoorebeke, and Veerle Cnudde. Real-time visualization of $\mathrm{h}$ aines jumps in sandstone with laboratory-based microcomputed tomography. Water Resources Research, 51(10):8668-8676, 2015. 1

[9] Hu Chen, Yi Zhang, Yunjin Chen, Junfeng Zhang, Weihua Zhang, Huaiqiang Sun, Yang Lv, Peixi Liao, Jiliu Zhou, and Ge Wang. LEARN: Learned experts' assessment-based reconstruction network for sparse-data CT. IEEE transactions on medical imaging, 37(6):1333-1347, 2018. 2

[10] Zhiqiang Chen, Xin Jin, Liang Li, and Ge Wang. A limitedangle CT reconstruction method based on anisotropic TV minimization. Physics in Medicine \& Biology, 58(7):2119, 2013. 2

[11] Veerle Cnudde and Matthieu Nicolaas Boone. Highresolution X-ray computed tomography in geosciences: A review of the current technology and applications. EarthScience Reviews, 123:1-17, 2013. 1

[12] Zhiming Cui, Changjian Li, and Wenping Wang. ToothNet: Automatic tooth instance segmentation and identification from cone beam CT images. In Proc. CVPR, pages 6368-6377, 2019. 1

[13] Vincent De Andrade, Alex Deriy, Michael J Wojcik, Doga Gürsoy, Deming Shu, Kamel Fezzaa, and Francesco
De Carlo. Nanoscale 3D imaging at the advanced photon source. SPIE Newsroom, 10(2.1201604):006461, 2016. 1

[14] P De Santis and F Gori. On an iterative method for superresolution. Optica Acta: International Journal of Optics, 22(8):691-695, 1975. 2

[15] Lee A Feldkamp, Lloyd C Davis, and James W Kress. Practical cone-beam algorithm. Josa a, 1(6):612-619, 1984. 2

[16] José-Jesús Fernández and Sam Li. An improved algorithm for anisotropic nonlinear diffusion for denoising cryotomograms. Journal of structural biology, 144(1-2):152161, 2003. 3

[17] Johannes Feulner, S Kevin Zhou, Martin Huber, Joachim Hornegger, Dorin Comaniciu, and Alexander Cavallaro. Lymph node detection in 3-D chest CT using a spatial prior probability. In Proc. CVPR, pages 2926-2932. IEEE, 2010. 1

[18] Irena Galić, Joachim Weickert, Martin Welk, Andrés Bruhn, Alexander Belyaev, and Hans-Peter Seidel. Image compression with anisotropic diffusion. Journal of Mathematical Imaging and Vision, 31(2-3):255-269, 2008. 3

[19] Mariana-Iuliana Georgescu, Radu Tudor Ionescu, and Nicolae Verga. Convolutional neural networks with intermediate loss for 3D super-resolution of CT and MRI scans. IEEE Access, 8:49112-49124, 2020. 3

[20] RW Gerchberg. Super-resolution through error energy reduction. Optica Acta: International Journal of Optics, 21(9):709-720, 1974. 2

[21] Richard Gordon, Robert Bender, and Gabor $\mathrm{T}$ Herman. Algebraic reconstruction techniques (ART) for threedimensional electron microscopy and X-ray photography. Journal of theoretical Biology, 29(3):471-481, 1970. 2

[22] Yeisson Gutiérrez, David Ott, Mareike Töpperwien, Tim Salditt, and Christoph Scherber. X-ray computed tomography and its potential in ecological research: A review of studies and optimization of specimen preparation. Ecology and evolution, 8(15):7717-7732, 2018. 1

[23] Janka Hatvani, Adrian Basarab, Jean-Yves Tourneret, Miklós Gyöngy, and Denis Kouamé. A tensor factorization method for 3-D super resolution with application to dental CT. IEEE Trans. Med. Img., 38(6):1524-1531, 2018. 3

[24] Janka Hatvani, András Horváth, Jérôme Michetti, Adrian Basarab, Denis Kouamé, and Miklós Gyöngy. Deep learning-based super-resolution applied to dental computed tomography. IEEE Transactions on Radiation and Plasma Medical Sciences, 3(2):120-128, 2018. 3

[25] Rob Heylen, Georg Schramm, Paul Suetens, and Johan Nuyts. 4D CBCT reconstruction with TV regularization on a dynamic software phantom. In 2019 IEEE Nuclear Science Symposium and Medical Imaging Conference (NSS/MIC), pages 1-3. IEEE. 2

[26] Dianlin Hu, Jin Liu, Tianling Lv, Qianlong Zhao, Yikun Zhang, Guotao Quan, Juan Feng, Yang Chen, and Limin Luo. Hybrid domain neural network processing for sparseview CT reconstruction. IEEE Transactions on Radiation and Plasma Medical Sciences, 2020. 2

[27] Jing Huang, Yunwan Zhang, Jianhua Ma, Dong Zeng, Zhaoying Bian, Shanzhou Niu, Qianjin Feng, Zhengrong 
Liang, and Wufan Chen. Iterative image reconstruction for sparse-view CT using normal-dose image induced total variation prior. PloS one, 8(11):e79709, 2013. 2

[28] Yawen Huang, Ling Shao, and Alejandro F Frangi. Simultaneous super-resolution and cross-modality synthesis of 3D medical images using weakly-supervised joint convolutional sparse coding. In Proc. CVPR, pages 6070-6079, 2017. 3

[29] Nick Janssens, Marijke Huysmans, and Rudy Swennen. Computed tomography 3D super-resolution with generative adversarial neural networks: Implications on unsaturated and two-phase fluid flow. Materials, 13(6):1397, 2020. 3

[30] Xin Jin, Liang Li, Zhiqiang Chen, Li Zhang, and Yuxiang Xing. Anisotropic total variation for limited-angle CT reconstruction. In IEEE Nuclear Science Symposuim \& Medical Imaging Conference, pages 2232-2238. IEEE, 2010. 2

[31] Hyunwoo Kim, Jeong-Hun Jang, and Ki-Sang Hong. Edgeenhancing super-resolution using anisotropic diffusion. In Proc. ICIP, volume 3, pages 130-133. IEEE, 2001. 3

[32] D Kroon and Cornelis H Slump. Coherence filtering to enhance the mandibular canal in cone-beam CT data. In IEEEEMBS Benelux Chapter Symposium, volume 11, pages 4041, 2009. 5

[33] Amine Laghrib, Aissam Hadri, and Abdelilah Hakim. An edge preserving high-order PDE for multiframe image superresolution. Journal of the Franklin Institute, 356(11):58345857, 2019. 3

[34] Zhengchun Liu, Tekin Bicer, Rajkumar Kettimuthu, Doga Gursoy, Francesco De Carlo, and Ian Foster. TomoGAN: low-dose synchrotron x-ray tomography with generative adversarial networks: discussion. JOSA A, 37(3):422-434, 2020. 2

[35] Adriënne M Mendrik, Evert-Jan Vonken, Annemarieke Rutten, Max A Viergever, and Bram van Ginneken. Noise reduction in computed tomography scans using 3-D anisotropic hybrid diffusion with continuous switch. IEEE Trans. Med. Img., 28(10):1585-1594, 2009. 3

[36] Domingo Mery, Daniel Saavedra, and Mukesh Prasad. X-ray baggage inspection with computer vision: A survey. IEEE Access, 8:145620-145633, 2020. 1

[37] Kamal Nasrollahi and Thomas B Moeslund. Superresolution: a comprehensive survey. Machine vision and applications, 25(6):1423-1468, 2014. 2

[38] Viktor V Nikitin, Marcus Carlsson, Fredrik Andersson, and Rajmund Mokso. Four-dimensional tomographic reconstruction by time domain decomposition. IEEE Trans. Comp. Img., 5(3):409-419, 2019. 2

[39] Shanzhou Niu, Yang Gao, Zhaoying Bian, Jing Huang, Wufan Chen, Gaohang Yu, Zhengrong Liang, and Jianhua Ma. Sparse-view x-ray CT reconstruction via total generalized variation regularization. Physics in Medicine \& Biology, 59(12):2997, 2014. 2

[40] Ozan Oktay, Wenjia Bai, Matthew Lee, Ricardo Guerrero, Konstantinos Kamnitsas, Jose Caballero, Antonio de Marvao, Stuart Cook, Declan O'Regan, and Daniel Rueckert. Multi-input cardiac image super-resolution using convolutional neural networks. In International conference on medical image computing and computer-assisted intervention, pages 246-254. Springer, 2016. 2
[41] Xiaochuan Pan, Emil Y Sidky, and Michael Vannier. Why do commercial CT scanners still employ traditional, filtered back-projection for image reconstruction? Inverse problems, 25(12):123009, 2009. 2

[42] Yaniv Romano, Michael Elad, and Peyman Milanfar. The little engine that could: Regularization by denoising (RED). SIAM Journal on Imaging Sciences, 10(4):1804-1844, 2017. 2, 3

[43] Kuldeep Singh, Anubhav Gupta, and Rajiv Kapoor. Fingerprint image super-resolution via ridge orientation-based clustered coupled sparse dictionaries. Journal of Electronic Imaging, 24(4):043015, 2015. 2

[44] Chao Tang, Wenkun Zhang, Ziheng Li, Ailong Cai, Linyuan Wang, Lei Li, Ningning Liang, and Bin Yan. Projection super-resolution based on convolutional neural network for computed tomography. In 15th International Meeting on Fully Three-Dimensional Image Reconstruction in Radiology and Nuclear Medicine, volume 11072, page 1107233. International Society for Optics and Photonics, 2019. 3

[45] Dang Thanh, Prasath Surya, et al. A review on CT and X-ray images denoising methods. Informatica, 43(2), 2019. 1

[46] Jing Tian and Kai-Kuang Ma. A survey on super-resolution imaging. Signal, Image and Video Processing, 5(3):329-342, 2011. 2

[47] Alina Toma, Loïc Denis, Bruno Sixou, Jean-Baptiste Pialat, and Françoise Peyrin. Total variation super-resolution for 3D trabecular bone micro-structure segmentation. In 2014 22nd European Signal Processing Conference (EUSIPCO), pages 2220-2224. IEEE, 2014. 3

[48] Wim Van Aarle, Kees Joost Batenburg, Gert Van Gompel, Elke Van de Casteele, and Jan Sijbers. Super-resolution for computed tomography based on discrete tomography. IEEE Trans. Image Proc., 23(3):1181-1193, 2014. 1, 3

[49] Bram Van Ginneken, BM Ter Haar Romeny, and Max A Viergever. Computer-aided diagnosis in chest radiography: a survey. IEEE Trans. Med. Img., 20(12):1228-1241, 2001. 1

[50] Gert Van Gompel, K Joost Batenburg, Elke Van de Casteele, Wim Van Aarle, and Jan Sijbers. A discrete tomography approach for superresolution micro-CT images: application to bone. In 2010 IEEE International Symposium on Biomedical Imaging: From Nano to Macro, pages 816-819. IEEE, 2010. 3

[51] Bert Vandeghinste, Bart Goossens, Jan De Beenhouwer, Aleksandra Pizurica, Wilfried Philips, Stefaan Vandenberghe, and Steven Staelens. Split-Bregman-based sparseview $\mathrm{CT}$ reconstruction. In 11th International meeting on Fully Three-Dimensional Image Reconstruction in Radiology and Nuclear Medicine (Fully 3D 11), pages 431-434, 2011. 2

[52] Ting Wang, Katsuhiro Nakamoto, Heye Zhang, and Huafeng Liu. Reweighted anisotropic total variation minimization for limited-angle CT reconstruction. IEEE Transactions on $\mathrm{Nu}$ clear Science, 64(10):2742-2760, 2017. 5

[53] Yuzhu Wang, Sheik S Rahman, and Christoph H Arns. Super resolution reconstruction of $\mu$-CT image of rock sample using neighbour embedding algorithm. Physica A: Statistical Mechanics and its Applications, 493:177-188, 2018. 3 
[54] Zhihao Wang, Jian Chen, and Steven CH Hoi. Deep learning for image super-resolution: A survey. IEEE Trans. PAMI, 2020. 2

[55] Joachim Weickert. Theoretical foundations of anisotropic diffusion in image processing. In Theoretical foundations of computer vision, pages 221-236. Springer, 1996. 3

[56] Joachim Weickert. Coherence-enhancing diffusion filtering. International journal of computer vision, 31(2-3):111-127, 1999. 3

[57] Joachim Weickert and Hanno Scharr. A scheme for coherence-enhancing diffusion filtering with optimized rotation invariance. Journal of Visual Communication and Image Representation, 13(1-2):103-118, 2002. 3, 5

[58] Daiqin Yang, Zimeng Li, Yatong Xia, and Zhenzhong Chen. Remote sensing image super-resolution: Challenges and approaches. In 2015 IEEE international conference on digital signal processing (DSP), pages 196-200. IEEE, 2015. 2

[59] Haichao Yu, Ding Liu, Honghui Shi, Hanchao Yu, Zhangyang Wang, Xinchao Wang, Brent Cross, Matthew Bramler, and Thomas S Huang. Computed tomography super-resolution using convolutional neural networks. In Proc. ICIP, pages 3944-3948. IEEE, 2017. 3

[60] Guangming Zang, Mohamed Aly, Ramzi Idoughi, Peter Wonka, and Wolfgang Heidrich. Super-resolution and sparse view CT reconstruction. In Proc. ECCV, pages 137-153, 2018. 1, 2, 3, 5, 7

[61] Guangming Zang, Ramzi Idoughi, Ran Tao, Gilles Lubineau, Peter Wonka, and Wolfgang Heidrich. Space-time tomography for continuously deforming objects. ACM Trans. Graph., 37(4):36, 2018. 1, 2

[62] Guangming Zang, Ramzi Idoughi, Ran Tao, Gilles Lubineau, Peter Wonka, and Wolfgang Heidrich. Warp-and-project tomography for rapidly deforming objects. ACM Trans. Graph., 38(4), 2019. 1, 2

[63] Yu Zhang, Guorong Wu, Pew-Thian Yap, Qianjin Feng, Jun Lian, Wufan Chen, and Dinggang Shen. Reconstruction of super-resolution lung 4D-CT using patch-based sparse representation. In Proc. CVPR, pages 925-931. IEEE, 2012. 2

[64] Shuang Zhao, Wenzel Jakob, Steve Marschner, and Kavita Bala. Building volumetric appearance models of fabric using micro ct imaging. ACM Trans. Graph., 30(4):1-10, 2011. 1 